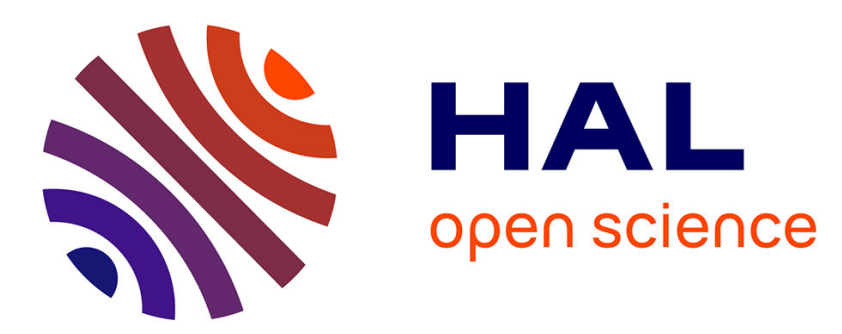

\title{
Finite-time stabilization of underactuated mechanical systems in the presence of uncertainties: application to the cart-pole system
}

Jawhar Ghommam, Ahmed Chemori, Faiçal Mnif

\section{- To cite this version:}

Jawhar Ghommam, Ahmed Chemori, Faiçal Mnif. Finite-time stabilization of underactuated mechanical systems in the presence of uncertainties: application to the cart-pole system. The Inverted Pendulum in Control Theory and Robotics: From Theory to New Innovations, Chapter 8, pp.165-187, 2017, 978-1-78561-320-3. 10.1049/PBCE111E_ch8. lirmm-01891593

\section{HAL Id: lirmm-01891593 \\ https://hal-lirmm.ccsd.cnrs.fr/lirmm-01891593}

Submitted on 9 Oct 2018

HAL is a multi-disciplinary open access archive for the deposit and dissemination of scientific research documents, whether they are published or not. The documents may come from teaching and research institutions in France or abroad, or from public or private research centers.
L'archive ouverte pluridisciplinaire HAL, est destinée au dépôt et à la diffusion de documents scientifiques de niveau recherche, publiés ou non, émanant des établissements d'enseignement et de recherche français ou étrangers, des laboratoires publics ou privés. 
Chapter 8

\title{
Finite-time stabilization of underactuated
} mechanical systems in the presence of uncertainties: application to the cart-pole system

\author{
Jawhar Ghommam ${ }^{1}$, Ahmed Chemori ${ }^{2}$, and Faiçal Mnif ${ }^{3}$
}

\subsection{Introduction}

Underactuated mechanical systems $[13,14,28]$ are those systems with less control inputs than generalized coordinates (called also degrees of freedom), i.e. they have unactuated generalized coordinates. For such systems, the unactuated generalized coordinates may indirectly be controlled by the actuated coordinates through the dynamic coupling, inherent to these systems [8]. This coupling is often nonlinear, resulting in generally nonintegrable dynamic constraints and therefore second-order nonholonomic.

Many examples of such systems exist, and mainly in robotics. They include, among others, Inverted pendulums [16,26,27], underactuated robot manipulators [6], gymnast robots and particularly the acrobot [42] and the pendubot [32], undersea vehicles [15], PVTOL (Planar Vertical Takeoff and Landing) aircrafts [36] and mobile robots [18].

Underactuation in these mechanical systems is generally introduced intentionally at the design level to reduce the manufacturing cost, the weight and/or failure rate; consequently, the obtained systems may be able to perform complex tasks with a reduced number of actuators. The source of underactuation may also be unintentionally due to failure of one or more actuators in a fully actuated system.

Underactuated systems may require new approaches/techniques to design effective control strategies; therefore, they constitute a good framework for nonlinear control problems regarding both theoretical and practical aspects. For these reasons, they are attracting more and more attention of researchers from nonlinear control community as well as from many research fields in robotics.

In the literature, many research efforts have been made in control aspects [31-33,40,42-44], giving rise to multiple control solutions; however, control problem of such systems is still open.

\footnotetext{
${ }^{1}$ National Institute of Applied Sciences and Technology (INSAT), Tunisia

${ }^{2}$ Laboratoire d'Informatique, de Robotique et de Microélectronique de Montpellier (LIRMM), France

${ }^{3}$ College of Engineering, Sultan Qaboos University, Sultanate of Oman
} 


\section{The inverted pendulum in control theory and robotics}

For instance in [9], a technique of pseudolinearization using spline functions is proposed to control the acrobot underactuated system. In [43], a design technique combining partial feedback linearization and Lyapunov methods based on saturation functions, switching and energy shaping has been proposed. In [44], the author discuss a survey on some existing results such as geometric nonlinear control and passivity-based control for stabilization and tracking control of such systems. Three control algorithms have been proposed in [31] for an underactuated two-link robot, namely an optimal LQ controller, a partial feedback-linearizing regulator and a sliding mode controller. In [32], the testbed is also a two-link underactuated robot but the actuator is located at the base (in contrast to the one proposed in [31] where the actuator is located at the joint between the two links), and the authors propose a method based on partial feedback linearization [41], nilpotent approximation and an iterative stabilization procedure. Roughly speaking, they propose an open-loop controller able to steer the system closer to the desired equilibrium point in finite time, and apply it iteratively, which provides a robust exponential convergence to the equilibrium. In [33], the authors propose to study the nonlinear behavior of the same robot proposed in [32] through a graphical tool based on Poincaré map. The study in [40] concerns underactuated mechanical systems with several actuated degrees of freedom and a single unactuated degree of freedom, where a discontinuous nonlinear feedback controller is proposed, allowing the closed-loop equilibrium at the origin to be globally attractive. In [46], a generalized predictive controller has been proposed, based on the linearized dynamics of the system. In [10], a nonlinear predictive controller is proposed for both stabilization and stable-limit cycle generation with an application to the ECP 505 inverted pendulum. In [45], a technique based on switching and saturation control is proposed. In [11], a passivity-based approach has been proposed to control the inertia wheel inverted pendulum. In [17], the technique of virtual holonomic constraints has been proposed with an application to the pendubot for generation of periodic motions, and to the Inertia Wheel Pendulum in [16]. In [3], the proposed control techniques are based on partial feedback linearization, combined with the optimization of the reference trajectories to stabilize the internal dynamics of the system. In [4] and [5], a dual-model-free control technique is proposed. The control architecture includes two model-free controllers, the first one is used to track the reference trajectories on the actuated degrees of freedom of the system, whereas the second one is used to stabilize the internal dynamics. In [26], a technique based on Riccati and nonlinear control is proposed for inverted pendulums. Output feedback control with an extended high gain observer was recently proposed in [29].

To deal with model uncertainties, a time-scale approach along with the Lyapunov design has been proposed in [39]. The authors in [35] used a sliding mode technique for the cart-pole system to stabilize the system in presence of disturbances. Model uncertainties however have not been tackled in this work. In [1], disturbances and model uncertainties have been fully considered in a technique that involves a backstepping procedure combined with sliding mode, applied to the inverted pendulum system after the system was converted into a normal form. Integral sliding mode control was also applied in [47] to deal with uncertainties in the two-wheeled mobile 
inverted pendulum. The control law being designed is based on the linearized system dynamics which resulted in a small region of attraction for the equilibrium. The problem becomes more challenging when ever nonparametric uncertainties and unknown time-varying disturbances are considered in the design of effective and robust control law for the underactuated system.

An important research issue which has attracted the attention of the control community is the finite-time convergence and finite-time stabilization [2,7,19-21,24], that ensures faster convergence rate, better disturbance rejection and robustness against uncertainty. Although myriad approaches have been proposed in the literature that tackle the problem of finite-time stabilization, they are not effective in dealing with time-varying and state-depending uncertainties. Few attempts have been concentrated in the design of adaptive schemes [22,23] to resolve the problem of state dependent and time-varying uncertainties. However, the adaptive scheme has only been applied to a class of $p$-normal forms [12]. To the best of the authors' knowledge, few have dealt with finite-time stabilization of underactuated systems subject to time-varying and state-dependent uncertainties. This chapter focuses on the design of a novel nonlinear controller for a class of underactuated system; we would like to introduce a new strategy that addresses an adaptive robust finite-time stabilization law derived from adding a power integrator (API) [37] technique to a class of single input dynamics described as a normal form system [34]. The idea is to generate coordinate transformations that decouple the underactuated dynamics into an upper-triangular form allowing for only one control input governing the whole system to be designed, whereby a continuous finite-time stabilizing control law is derived from the API technique, and an adaptive compensator is proposed to compensate for the matched and unmatched uncertainties collected throughout the steps of the design. As a consequence, the resulting control law is able to stabilize the underactuated mechanical system in a finite time.

The reminder of this chapter is organized as follows. Section 8.2 discusses the Lagrangian model of mechanical underactuated systems and the coordinate transformation that transforms this model into a normal-form representation. Section 8.3 formulates the finite-time stabilization problem for the $n$ th-order underactuated system. Section 8.4 gives a background on finite-time stability and provides some useful lemmas needed throughout the paper. The control design is developed in Section 8.5. Simulation results with an application to the cart-pole system are presented and discussed in Section 8.6. Finally, Section 8.7 draws a conclusion for this chapter.

\subsection{Dynamic modeling of underactuated mechanical systems: Lagrangian formalism}

The Lagrangian formalism is one among the powerful mathematical tools based on the vibrational method to model a large class of mechanical systems. Underactuated mechanical systems are branches of this class. They can be modeled using the EulerLagrange equations. Let $Q$ be an $n$-dimensional configuration manifold, and $q \in Q$ is a 
vector of generalized coordinates. The Euler-Lagrange equations for any mechanical system of $n$ degrees of freedom (DOF) are given by [34]

$$
\frac{d}{d t} \frac{\partial L(q, \dot{q})}{\partial \dot{q}}-\frac{\partial L(q, \dot{q})}{\partial q}=B(q) u
$$

where $u \in \mathbb{R}^{m}$ and $B(q)$ is the external forces matrix. $L(q, \dot{q})$ is the Lagrangian associated to the mechanical system and expressed by the difference between kinetic and potential energies:

$$
L(q, \dot{q})=K-V=\frac{1}{2} \dot{q}^{\top} M(q) \dot{q}-V(q)
$$

where $K$ is the kinetic energy, $V(q)$ denotes the potential energy and $M(q)=M^{\top}(q) \in$ $\mathbb{R}^{n \times n}$ is a positive definite inertia matrix. Let $m_{i k} \in \mathbb{R}^{+}$denote the entries of the inertia matrix $M$ with $i=1, \ldots, n$ and $k=1, \ldots, n$. Based on (8.1) and (8.2), the equations of motion can be deduced as follows:

$$
\sum_{k} m_{i k}(q) \ddot{q}_{k}+\sum_{i, k} \Gamma_{i k}^{\ell}(q) \dot{q}_{i} \dot{q}_{k}+g_{i}(q)=e_{\ell}^{\top} B(q) u, \quad \ell=1, \ldots, n
$$

where $e_{\ell}$ is the $\ell$ th standard basis in $R^{n}, g_{i}=\partial_{q i} V(q)$, and $\Gamma_{i k}^{\ell}(q)$ is the Christoffel symbol defined by

$$
\Gamma_{i k}^{\ell}(q)=\frac{1}{2}\left(\frac{\partial m_{\ell k}(q)}{\partial q_{i}}+\frac{\partial m_{\ell i}(q)}{\partial q_{k}}-\frac{\partial m_{i k}(q)}{\partial q_{\ell}}\right)
$$

In a matrix form, (8.3) can be rewritten as

$$
M(q) \ddot{q}+C(q, \dot{q}) \dot{q}+G(q)=B(q) u
$$

The term $C(q, \dot{q})$ includes two distinct terms, in particular, the first term $q_{i} \dot{q}_{k}$ involves the centrifugal forces $(i=k)$, and the second is the Coriolis forces for $(i \neq k)$, and $G(q)$ represents the gravity vector. It is interesting to point out that the mechanical system described by (8.5) satisfies the skew symmetric property of the matrix $\dot{M}(q)-$ $2 C(q, \dot{q})$.

It is important to distinguish two categories of mechanical systems governed by the equation of motion (8.5). A mechanical system described by (8.5) is said to be fully actuated if $m=n$, which is equivalent to say that the matrix $B(q) \in R^{n \times m}$ is invertible. In other words, it can be roughly inferred that the mechanical system is fully actuated, if the number of actuators is equal to the dimension of its configuration manifold. On the other hand, if $m<n$, the mechanical system is said to be underactuated, which is equivalent to have fewer independent inputs than the number of degree of freedom to be controlled. This implies that the matrix $B(q)$ becomes noninvertible and consequently the underactuated mechanical system as opposed to fully actuated systems is not exact feedback linearizable. Because the number of inputs is less than the number of degree of freedom, the controllability of the mechanical underactuated system is not always satisfied. In this chapter, however, we will assume that the 
considered mechanical systems are controllable. Furthermore, we suppose that the equation of motion (8.5) can be rearranged so that the matrix $B(q)$ can be written as:

$$
B(q)=\left[\begin{array}{c}
\mathbf{0}_{(n-m) \times m} \\
\mathbf{I}_{m}
\end{array}\right]
$$

where the first $n-m$ equations of (8.5) represent the unactuated dynamics while the last $m$ equations are the actuated dynamics. The generalized coordinates $q$ can further be partitioned as follows:

$$
q=\left[\begin{array}{l}
q_{u} \\
q_{a}
\end{array}\right]
$$

where $q_{u} \in \mathbb{R}^{(n-m)}$ is the vector of unactuated configuration variables, and $q_{a} \in \mathbb{R}^{m}$ is the vector of actuated configuration variables. Then, it is straightforward to rewrite the equation of motion (8.5) as follows:

$$
\left[\begin{array}{ll}
m_{11}(q) & m_{12}(q) \\
m_{21}(q) & m_{22}(q)
\end{array}\right]\left[\begin{array}{l}
\ddot{q}_{u} \\
\ddot{q}_{a}
\end{array}\right]+\left[\begin{array}{ll}
c_{11}(q) & c_{12}(q) \\
c_{21}(q) & c_{22}(q)
\end{array}\right]\left[\begin{array}{l}
\dot{q}_{u} \\
\dot{q}_{a}
\end{array}\right]+\left[\begin{array}{l}
g_{1}(q) \\
g_{2}(q)
\end{array}\right]=\left[\begin{array}{c}
\mathbf{0} \\
\mathbf{u}_{2}
\end{array}\right]
$$

The partitioned equation of motion (8.8) can also be represented in component form as follows:

$$
\begin{aligned}
& m_{11}(q) \ddot{q}_{u}+m_{12}(q) \ddot{q}_{a}+h_{1}(q, \dot{q})=\mathbf{0} \\
& m_{21}(q) \ddot{q}_{u}+m_{22}(q) \ddot{q}_{a}+h_{2}(q, \dot{q})=\mathbf{u}_{2}
\end{aligned}
$$

where $h_{1}(q, \dot{q})=c_{11}(q) \dot{q}_{u}+c_{12}(q) \dot{q}_{a}+g_{1}(q)$ and $h_{2}(q, \dot{q})=c_{21}(q) \dot{q}_{u}+c_{22}(q) \dot{q}_{a}+$ $g_{2}(q)$. It is worth to notice that there is no control input in (8.9); therefore, it is not possible to feedback linearize the system (8.9) and (8.10). It is however possible to partially feedback linearize the system using a change of control. The procedure of linearization of the actuated dynamics is called collocated partial linearization which is credited to Spong [44] and consists in finding a global invertible change of control of the form $u_{2}=\alpha(q) v+\beta(q, \dot{q})$ that partially linearizes the dynamics (8.8) such as, after partial linearization, the new control $v$ appears in the nonlinear-subsystem dynamics as well as in the linearized subsystem dynamics as follows:

$$
\begin{aligned}
& \ddot{q}_{u}=f_{1}(q, \dot{q})+g_{1}(q, \dot{q}) v \\
& \ddot{q}_{a}=v
\end{aligned}
$$

It can be observed that the unactuated part (8.11) is still complex, while the actuated part (8.12) is linearized into a double-integrator dynamics. The new control input $v$ is present in both subsystems, which largely increases the difficulty in the control design. The author in [34] introduced a global change of coordinates that decouples these two subsystems and transforms the dynamics of the system into a cascaded form called the normal form, where the control input of the original actuated subsystem does not appear in the unactuated subsystem. This significantly simplifies the control design for the underactuated mechanical system. However, with this approach, there is an inherent requirement that the model parameters of the mechanical system must be perfectly known to be able to design controller for the transformed normal form. 


\section{The inverted pendulum in control theory and robotics}

Due to lack of knowledge on the system parameters and the inability to measure the whole system state, the controller has to cope with these uncertainties. This situation justifies the need for the development of a global transformation that decouples the underactuated mechanical system without passing by the partial feedback linearization of the system.

Consider the dynamic (8.9), resolve for $\ddot{q}_{u}$, it gives

$$
\ddot{q}_{u}=-m_{11}(q)^{-1}\left(m_{12}(q) \ddot{q}_{a}+h_{1}(q, \dot{q})\right)
$$

Substituting (8.13) into (8.10), after some manipulation, we obtain

$$
\ddot{q}_{a}=f_{3}(q, \dot{q})+b_{3}(q) \mathbf{u}_{2}
$$

Again substituting the resulting dynamics (8.14) into the dynamics of (8.10), then solving for $\ddot{q}_{u}$ leads to the following dynamics:

$$
\ddot{q}_{u}=f_{4}(q, \dot{q})+b_{4}(q) \mathbf{u}_{2}
$$

where $f_{3}(q, \dot{q})=\left(m_{22}-m_{21} m_{11}^{-1} m_{12}\right)^{-1}\left[m_{21} m_{11}^{-1} h_{1}(q, \dot{q})-h_{2}(q, \dot{q})\right]=, b_{3}(q)=\left(m_{22}-\right.$ $\left.m_{21} m_{11}^{-1} m_{12}\right)^{-1}, f_{4}(q, \dot{q})=-m_{11}^{-1} m_{12} f_{4}\left(q, \dot{q}-m_{11}^{-1} h_{1}(q, \dot{q})\right)$ and $b_{4}(q)=-m_{11}^{-1} m_{12} b_{3}(q)$. Regrouping the dynamic (8.14) and (8.15), one can obtain a state space representation of the system as follows:

$$
\begin{aligned}
& \dot{x}_{1}=x_{2} \\
& \dot{x}_{2}=f_{3}(q, \dot{q})+b_{3}(q) \mathbf{u}_{2} \\
& \dot{x}_{3}=x_{4} \\
& \dot{x}_{4}=f_{4}(q, \dot{q})+b_{4}(q) \mathbf{u}_{2}
\end{aligned}
$$

where the state space vector $x$ has been defined as $\left[x_{1}, x_{2}, x_{3}, x_{4}\right]^{\top}=\left[q_{u}, \dot{q}_{u}, q_{a}, \dot{q}_{a}\right]^{\top}$. Note the structural difference between (8.12), (8.11) and (8.16) is that the actuated dynamics in (8.12) is a double integrator, while in (8.16), the actuated dynamics has some nonlinearities.

To decouple the nonlinear system represented by (8.16), we may use the global transformation proposed in [34] as follows:

$$
\begin{aligned}
& z_{1}=x_{1}-\int_{0}^{x_{3}} \frac{b_{3}(s)}{b_{4}(s)} d s, \\
& z_{2}=x_{2}-\frac{b_{3}(x)}{b_{4}(x)}, \\
& z_{3}=x_{3} \\
& z_{4}=x_{4}
\end{aligned}
$$


The equation dynamics in the $Z$-space is found by taking the time derivative of (8.17), leading to

$$
\begin{aligned}
& \dot{z}_{1}=z_{2}+\frac{b_{3}(z)}{b_{4}(z)} z_{4}-\frac{d}{d t} \int_{0}^{z_{3}} \frac{b_{3}(s)}{b_{4}(s)} d s \\
& \dot{z}_{2}=f_{3}(z)-\frac{b_{3}(z)}{b_{4}(z)} f_{4}(z)-\frac{d}{d t}\left(\frac{b_{3}(z)}{b_{4}(z)}\right) z_{4} \\
& \dot{z}_{3}=z_{4} \\
& \dot{z}_{4}=f_{4}(z)+b_{4}(z) \mathbf{u}_{2}
\end{aligned}
$$

Clearly, the dynamics of the underactuated system in $Z$-space, is in a special cascade form with only one control input appearing in the last subsystem. To ease the representation, let us define $d_{1}(z)=\frac{b_{3}(z)}{b_{4}(z)} z_{4}-\frac{d}{d t} \int_{0}^{z_{3}} \frac{b_{3}(s)}{b_{4}(s)} d s, d_{2}(z)=-z_{3}+f_{3}(z)-\frac{b_{3}(z)}{b_{4}(z)} f_{4}(z)-$ $\frac{d}{d t}\left(\frac{b_{3}(z)}{b_{4}(z)}\right) z_{4}, d_{3}(z)=0$, then the system (8.18) can be rewritten as

$$
\begin{aligned}
& \dot{z}_{1}=z_{2}+d_{1}(z) \\
& \dot{z}_{2}=z_{3}+d_{2}(z) \\
& \dot{z}_{3}=z_{4}+d_{3}(z) \\
& \dot{z}_{4}=f_{4}(z)+b_{4}(z) \mathbf{u}_{2}
\end{aligned}
$$

where $d_{1}(z), d_{2}(z)$ and $d_{3}(z)$ are regarded as unmatched uncertainties to be compensated by the control $\mathbf{u}_{2}$. In a similar manner, an $n$ th-order underactuated system can be represented in the state space form as

$$
\begin{aligned}
\dot{x}_{1} & =x_{2} \\
\dot{x}_{2} & =f_{3}(q, \dot{q})+b_{3}(q) \mathbf{u} \\
\dot{x}_{3} & =x_{4} \\
\dot{x}_{4} & =f_{4}(q, \dot{q})+b_{4}(q) \mathbf{u} \\
& \vdots \\
\dot{x}_{n-1} & =x_{n} \\
\dot{x}_{n} & =f_{n}(q, \dot{q})+b_{n}(q) \mathbf{u}
\end{aligned}
$$

To decouple the $n$th order system (8.20), the global transformation as proposed in [34] can be extended to $n$th order recursively as follows:

$$
\begin{aligned}
& z_{1}=x_{1}-\int_{0}^{x_{3}} \frac{b_{3}(s)}{b_{4}(s)} d s, \\
& z_{2}=x_{2}-\frac{b_{3}(x)}{b_{4}(x)} \\
& z_{3}=x_{3}-\int_{0}^{x_{5}} \frac{b_{4}(s)}{b_{5}(s)} d s
\end{aligned}
$$


172 The inverted pendulum in control theory and robotics

$$
\begin{aligned}
z_{4} & =x_{4}-\frac{b_{4}(x)}{b_{5}(x)} \\
& \vdots \\
z_{n-3} & =x_{n-3}-\int_{0}^{x_{n-1}} \frac{b_{n-2}(s)}{b_{n}(s)} d s \\
z_{n-2} & =x_{n-2}-\frac{b_{n-1}(x)}{b_{n}(x)} \\
z_{n-1} & =x_{n-1} \\
z_{n} & =x_{n}
\end{aligned}
$$

Hence, the system dynamics in $Z$-space can similarly be obtained, taking the time derivative of (8.21), we have

$$
\begin{aligned}
\dot{z}_{1} & =z_{2}+\frac{b_{3}(z)}{b_{4}(z)} z_{4}-\frac{d}{d t} \int_{0}^{z_{3}} \frac{b_{3}(s)}{b_{4}(s)} d s \\
\dot{z}_{2} & =f_{3}(z)-\frac{b_{3}(z)}{b_{4}(z)} f_{4}-\frac{d}{d t}\left(\frac{b_{3}(z)}{b_{4}(z)}\right) z_{4} \\
\dot{z}_{3} & =z_{4}+\frac{b_{4}(z)}{b_{6}(z)} z_{6}-\frac{d}{d t} \int_{0}^{z_{5}} \frac{b_{4}(s)}{b_{6}(s)} d s \\
\dot{z}_{4} & =f_{4}(z)-\frac{b_{4}(z)}{b_{6}(z)} f_{6}(z)-\frac{d}{d t}\left(\frac{b_{4}(z)}{b_{6}(z)}\right) z_{6} \\
& \vdots \\
\dot{z}_{n-3} & =z_{n-2}+\frac{b_{n-2}(z)}{b_{n}(z)} z_{n}-\frac{d}{d t} \int_{0}^{z_{n-1}} \frac{b_{n-2}(s)}{b_{n}(s)} d s \\
\dot{z}_{n-2} & =f_{n-2}(z)-\frac{b_{n-2}(z)}{b_{n}(z)} f_{n}-\frac{d}{d t}\left(\frac{b_{n-2}(z)}{b_{n}(z)}\right) z_{n} \\
\dot{z}_{n-1} & =z_{n} \\
\dot{z}_{n} & =f_{n}(z)+b_{n}(z) \mathbf{u}
\end{aligned}
$$

Let us now define the following transformation:

$$
\begin{aligned}
& d_{1}(z)=\frac{b_{3}(z)}{b_{4}(z)} z_{4}-\frac{d}{d t} \int_{0}^{z_{3}} \frac{b_{3}(s)}{b_{4}(s)} d s \\
& d_{2}(z)=-z_{3}+f_{3}(z)-\frac{b_{3}(z)}{b_{4}(z)} f_{4}-\frac{d}{d t}\left(\frac{b_{3}(z)}{b_{4}(z)}\right) z_{4}
\end{aligned}
$$




$$
\begin{aligned}
d_{n-3} & =\frac{b_{n-2}(z)}{b_{n}(z)} z_{n}-\frac{d}{d t} \int_{0}^{z_{n-1}} \frac{b_{n-2}(s)}{b_{n}(s)} d s \\
d_{n-2} & =-z_{n-1}+f_{n-2}(z)-\frac{b_{n-2}(z)}{b_{n}(z)} f_{n}-\frac{d}{d t}\left(\frac{b_{n-2}(z)}{b_{n}(z)}\right) z_{n} \\
d_{n-1} & =0 \\
d_{n} & =f_{n}(z)
\end{aligned}
$$

The dynamics in (8.23) can be similarly written in a standard form like in (8.18) as follows:

$$
\begin{aligned}
\dot{z}_{i} & =z_{i+1}+d_{i}(z), \quad 1 \leq i \leq n-1 \\
\dot{z}_{n} & =b_{n}(z) \mathbf{u}+d_{n}(z) \\
y & =h(z)
\end{aligned}
$$

where $d_{i}(z) \in \mathbb{R}, i=1,2, \ldots, n$ are regarded as matched and unmatched uncertainties. It can be noticed that by construction, the variation bounds of the uncertainties $d_{i}(z), i=1, \ldots, n$ are not assumed to be known and also cannot be assumed linearly parameterizable into a multiplication of a known regressor and a vector of unknown constant parameters. Also for the simplicity of the control design, let us consider the following two assumptions:

Assumption 8.1. The terms $d_{i}(z), i=1, \ldots, n$ are all unknown functions, whose bounds are also unknown.

Assumption 8.2. The uncertain control coefficient $b_{n}(z)$ is bounded as $b_{\min } \leq b_{n}(z) \leq$ $b_{\max }$. and $b_{n}(z)$ is sign-definite such that $\operatorname{sign}\left(b_{n}(z)\right)=+1$. Further, it is convenient to assume that $b_{n}(z) \neq 0$.

\subsection{Control problem formulation}

In this chapter, we consider a class of underactuated systems of the form (8.20), which can be transformed without partial feedback linearization and through the global change of coordinates (8.21) into the cascaded form (8.24). Where $z=$ $\left[z_{1}, z_{2}, \ldots, z_{n}\right]^{\top} \in \Omega$, where $\Omega$ is a compact set of $\mathbb{R}^{n}, u \in \mathbb{R}$ is the control input and $d_{i}(z) \in \mathbb{R}, i=1, \ldots, n-1$ are unmatched uncertainties, while $d_{n}$ is the matched uncertainty. Under Assumptions 8.1 and 8.2, the idea is to design the controller u such that the system state $z$ tracks in finite time the desired trajectory $z_{d}^{*}=$ $\left[z_{1 d}^{*}, z_{2 d}^{*}, \ldots, z_{n d}^{*}\right]^{\top} \in \Omega_{d}$ where $\Omega_{d}$ is a compact set of $\Omega$. This means that for any finite initial condition $z_{i}(0), i=1, \ldots, n$, there exists a finite time $T$ such that

$$
\lim _{t \rightarrow T}\left|z_{i}-z_{i d}^{*}\right|=0, \quad i=1, \ldots, n
$$

Remark 8.1. The control design satisfying the limit (8.25) guarantees stabilization in finite time of the underactuated mechanical system of the form (8.20) to a desired reference input $x_{d}$. In most of the cases, stabilization around the origin of the first state 
174 The inverted pendulum in control theory and robotics

$x_{1}$ is desired. The reference input for the Z-subsystem $z_{1 d}^{*}=0$ implies stabilization of the state variable $x_{1}$ to zero.

\subsection{Preliminaries}

In this section, a review of some basic concepts and lemma related to the concept of finite-time stability and the corresponding Lyapunov theorem are presented; they will be useful for most of the stability analysis of this chapter.

\subsubsection{Notions of finite stability}

Theorem 8.1. [7]: Consider the non-Lipschitz continuous autonomous system $\dot{x}=$ $f(x), f(0) \in \mathbb{R}$. Assume, there are $C^{1}$ function $V(x)$ defined on a neighborhood $D \subset \mathbb{R}$ of the origin, and real numbers $c>0$ and $0<\alpha<1$ such that

1. $V(x)$ is positive definite on $D$

2. $\dot{V}(x)+c V^{\alpha} \leq 0, \quad \forall x \in D$

Then, the origin $x$ is locally finite-time stable. If $D=\mathbb{R}$, and $V(x)$ is radially unbounded, then the origin $x=0$ is globally finite-time stable. Moreover, it can be verified that the settling time being dependent on the initial state $x(0)=x_{0}$ satisfies $T_{x}\left(x_{0}\right) \leq \frac{V\left(x_{0}\right)^{1-\alpha}}{c(1-\alpha)}$ for all $x_{0}$ in some open neighborhood of the origin.

Lemma 8.1. [37] For any $x_{i} \in \mathbb{R}, i=1, \ldots, n$ and $0<p \leq 1$, the following inequality holds $\left(\left|x_{1}\right|+\cdots+\left|x_{n}\right|\right)^{p} \leq\left|x_{1}\right|^{p}+\cdots+\left|x_{n}\right|^{p} \leq n^{1-p}\left(\left|x_{1}\right|+\cdots+\left|x_{n}\right|\right)^{p}$. When $0<p=\frac{p_{1}}{p_{2}} \leq 1$, where $p_{1}$ and $p_{2}$ are positive odd integers, then the following holds $\left|x^{p}-y^{p}\right| \leq 2^{1-p}|x-y|^{p}$, for any $x, y \in \mathbb{R}$.

Lemma 8.2. [37] For any $x \in \mathbb{R}, y \in \mathbb{R}, c>0, d>0$ and $\gamma(x, y)>0, a$ real-valued function, the following holds: $|x|^{c}|y|^{d} \leq(c \gamma(x, y) /(c+d))|x|^{c+d}+$ $\left(d \gamma(x, y)^{-c / d} /(c+d)\right)|y|^{c+d}$.

\subsection{Proposed control design}

The purpose of this section is to devise a control law that stabilizes the system (8.24) in finite time. Given the fact that the Z-system (8.24) contains unmatched uncertainty, traditional adaptive techniques like the backstepping technique [30] and the multiple-surface sliding [38] cannot be used, because the variation bounds of $d_{i}, i=1, \ldots, n$ are not assumed to be known. To solve the problem of finite-time stabilization with unmatched uncertainties, we propose a novel robust finite-time control scheme whereby a continuous recursive finite-time stabilizing control law is derived from the adding power integrator (API) technique [37] and an adaptive compensator. 
To develop the finite-time controller for (8.24), we first introduce the following tracking error $e_{1}$ and the virtual errors $e_{m}, m=2, \ldots, n$ as follows:

$$
e_{1}=z_{1}-z_{1 d}^{*}
$$

and

$$
e_{m}=z_{m}^{1 / q_{m}}-z_{m d}^{* 1 / q_{m}}, \quad m=2, \ldots, n
$$

where $q_{m}=(4 \ell n+3-2 m) /(4 \ell n+1)>0,\left(\ell \in \mathbb{Z}_{+}\right) ; z_{m}^{*}, m=2, \ldots, n$ is the virtual control given by $z_{m d}^{*}=-\beta_{m-1} e_{m-1}^{q_{m}}$ with $\beta_{m-1}$ being positive constant to be determined later. One main feature for the use of the parameter $\ell$ in the fraction power $q_{m}$ is that it allows adjusting the control precision and finite-time convergence of the state variables.

The design procedure consists in $n$ steps, throughout the $n-1$ steps, the virtual controllers $z_{(m-1) d}^{*}$ will be designed. Upon the completion of step $n$, the fast control term as well the adaptive compensator are designed.

Step 1: Consider the following Lyapunov candidate function:

$$
V_{1}(z)=\frac{1}{1+S} e_{1}^{1+S}
$$

where $S=(4 \ell n-1) /(4 \ell n+1)$. The time derivative of $(8.28)$ gives

$$
\begin{aligned}
\dot{V}_{1}(z) & =e_{1}^{S} \dot{e}_{1} \\
& =e_{1}^{S} z_{2}^{*}+e_{1}^{S}\left(z_{2}-z_{2 d}^{*}\right)+e_{1}^{S} d_{1}(z)
\end{aligned}
$$

By introducing the virtual control $z_{2 d}^{*}=-\beta_{1} e_{1}^{S}$, where $\beta_{1}$ is a design parameter, $\dot{V}_{1}(z)$ rewrites:

$$
\dot{V}_{1}(z)=-\beta_{1} e_{1}^{2 S}+e_{1}^{S}\left(z_{2}-z_{2 d}^{*}\right)+e_{1}^{S} d_{1}(z)
$$

Using Lemmas 8.1 and 8.2, the second term of (8.30) can be upper-bounded as follows:

$$
e_{1}^{S}\left(z_{2}-z_{2 d}^{*}\right) \leq 2^{1-S}\left|e_{2}\right|^{S}\left|e_{1}\right|^{S} \leq \frac{1}{2}\left|e_{1}\right|^{2 S}+C_{2}\left|e_{2}\right|^{2 S}
$$

where $C_{2}$ is a positive constant. In light of (8.31), the time derivative of $V_{1}(z)$ can be written as follows:

$$
\dot{V}_{1}(z) \leq-\beta_{1} e_{1}^{2 S}+\frac{1}{2}\left|e_{1}\right|^{2 S}+C_{2}\left|e_{2}\right|^{2 S}+e_{1}^{S} d_{1}(z)
$$

Step 2: According to [25], a $C^{1}$ and positive definite Lyapunov candidate function is constructed as

$$
V_{2}((z))=V_{1}(z)+W_{2}\left(z_{1}, z_{2}\right)
$$

with

$$
W_{2}\left(z_{1}, z_{2}\right)=\int_{z_{2 d}^{*}}^{z_{2}}\left(\chi^{\frac{1}{q_{2}}}-z_{2 d}^{* \frac{1}{q_{2}}}\right)^{1+S-q_{2}} d \chi
$$




\section{The inverted pendulum in control theory and robotics}

then it is easy to show that the Lyapunov function candidate $V_{2}(z)$ is positive definite and satisfies $V_{2}(z) \leq \max \left\{\frac{1}{1+S}, 2\right\}\left(e_{1}^{1+S}+e_{2}^{1+S}\right)$. Note that the function $W_{2}\left(z_{1}, z_{2}\right)$ has the following properties:

$$
\begin{aligned}
\frac{\partial W_{2}(.)}{\partial z_{2}} & =e_{2}^{1+S-q_{2}} \\
\frac{\partial W_{2}(.)}{\partial z_{1}} & =-\left(1+S-q_{2}\right) \frac{\partial z_{2 d}^{* \frac{1}{q_{2}}}}{\partial z_{1}} \int_{z_{2 d}^{*}}^{z_{2}}\left(\chi^{\frac{1}{q_{2}}}-z_{2 d}^{* \frac{1}{q_{2}}}\right)^{S-q_{2}} d \chi \\
& =-\frac{\partial z_{2 d}^{* \frac{1}{q_{2}}}}{\partial z_{1}}\left(z_{2}-z_{2 d}^{*}\right)
\end{aligned}
$$

Hence, the derivative of $W_{2}$ (.) can be computed as follows:

$$
\begin{aligned}
\dot{W}_{2}(.) & =e_{2} \dot{z}_{2}-\left(\left(z_{2}-z_{2 d}^{*}\right) \frac{\partial z_{2 d}^{* \frac{1}{q_{2}}}}{\partial z_{1}} \dot{z}_{1}\right) \\
& =e_{2}\left(z_{3}-z_{3 d}^{*}\right)+e_{2} z_{3}^{*}+e_{2} d_{2}(z)-\left(z_{2}-z_{2 d}^{*}\right) \frac{\partial z_{2 d}^{* \frac{1}{q_{2}}}}{\partial z_{1}} \dot{z}_{1}
\end{aligned}
$$

using the fact that

$$
\left|\left(z_{2}-z_{2 d}^{*}\right) \frac{\partial z_{2 d}^{* \frac{1}{q_{2}}}}{\partial z_{1}} \dot{z}_{1}\right| \leq 2^{1-q_{2}} e_{2}^{q_{2}}\left|\frac{\partial z_{2 d}^{* \frac{1}{q_{2}}}}{\partial z_{1}} z_{2}\right|+2^{1-q_{2}} e_{2}^{q_{2}}\left|\frac{\partial z_{2 d}^{* \frac{1}{q_{2}}}}{\partial z_{1}} d_{1}(z)\right|
$$

Let the first lumped unmatched uncertainty be $\tau_{1}=e_{2} d_{2}(z)+2^{1-q_{2}} \beta_{1}^{\frac{1}{q_{2}}} e_{2}^{q_{2}} d_{1}(z)$, it is then straightforward to obtain

$$
\dot{W}_{2} \leq e_{2}\left(z_{3}-z_{3 d}^{*}\right)+e_{2} z_{3 d}^{*}+2^{1-q_{2}} \beta^{\frac{1}{q_{2}}} e_{2}^{q_{2}}\left|z_{2}\right|+\tau_{1}
$$

According to Lemma 8.1, the third term of the right hand side of (8.39) can be bounded such that

$$
\left|z_{2}\right| \leq\left|e_{2}+z_{2 d}^{* \frac{1}{q_{2}}}\right|^{q_{2}} \leq\left|e_{2}\right|^{q_{2}}+\beta_{1}\left|e_{1}\right|^{q_{2}}
$$

which implies that

$$
\begin{aligned}
|e|_{2}^{q_{2}} 2^{1-q_{2}} \beta^{\frac{1}{q_{2}}}\left|z_{2}\right| & \leq 2^{1-q_{2}} \beta^{\frac{1}{q_{2}}}\left|e_{2}\right|^{2 q_{2}}+2^{1-q_{2}} \beta^{1+\frac{1}{q_{2}}}\left|e_{2}\right|^{q_{2}}\left|e_{1}\right|^{q_{2}} \\
& \leq 2^{2\left(1-q_{2}\right)-1} \beta^{2\left(1+\frac{1}{q_{2}}\right)}\left|e_{2}\right|^{2 q_{2}}+\frac{1}{2}\left|e_{1}\right|^{2 q_{2}}+2^{1-q_{2}} \beta^{\frac{1}{q_{2}}}\left|e_{2}\right|^{2 q_{2}} \\
& =\frac{1}{2}\left|e_{1}\right|^{2 q_{2}}+\bar{C}_{2}\left|e_{2}\right|^{2 q_{2}}
\end{aligned}
$$

where $\bar{C}_{2}=2^{2\left(1-q_{2}\right)} \beta^{2\left(1+\frac{1}{q_{2}}\right)}+2^{1-q_{2}} \beta^{\frac{1}{q_{2}}}$.

Combining (8.32), (8.39) and (8.41) yields the following:

$$
\dot{V}_{2}(.) \leq-\left(\beta_{1}-1\right)\left|e_{1}\right|^{2 S}+\left(C_{1}+\bar{C}_{2}\right)\left|e_{2}\right|^{2 S}+e_{3} z_{3 d}^{*}+e_{2}\left(z_{3}-z_{3 d}^{*}\right)+\tau_{1}
$$


At this stage, it is worth to note that $q_{3}=q_{2}-\frac{2}{4 \ell n+1}$, which implies that $q_{3}=$ $1+2 q_{2}=1+2 S$. Therefore, by selecting $\beta_{1}>n-1-\kappa$, where $\kappa>0$ is a design parameter, and the virtual control $z_{3 d}^{*}=-\beta_{2} z_{2 d}^{q_{3}}$ with $\beta_{2} \geq n-2+\kappa+C_{2}+\bar{C}_{2}$, we obtain

$$
\dot{V}_{2} \leq-(n-2+\kappa)\left|e_{1}\right|^{2 S}-(n-2+\kappa)\left|e_{2}\right|^{2 S}+e_{2}\left(z_{3}-z_{3 d}^{*}\right)+\tau_{1}
$$

Step $\boldsymbol{k}(\boldsymbol{k}:=\mathbf{3} \ldots \boldsymbol{n}-\mathbf{1})$ : We proceed to the derivation of the virtual control by using an inductive argument. Suppose at step $k-1$, that there exists a $C^{1}$ Lyapunov candidate function $V_{k-1}\left(z_{1}, \ldots, z_{k-1}\right)$, positive definite and verifies

$$
V_{k-1}(.) \leq \max \left\{\frac{1}{1+S}, 2\right\} \sum_{m=1}^{k-1} e_{m}^{1+S}
$$

such that

$$
\dot{V}_{k-1}(.) \leq-(n-k+1+\kappa) \sum_{m=1}^{k-1} e_{m}^{2 S}+e_{k-1}^{1+S-q_{k-1}}\left(z_{k}-z_{k d}^{*}\right)+\sum_{m=1}^{k-2} \tau_{m}
$$

Now let us claim that (8.44) and (8.45) hold as step $k$. To prove this claim, consider the following Lypunov candidate function:

$$
V_{k}\left(z_{1}, \ldots, z_{k}\right)=V_{k-1}(.)+W_{k}\left(z_{1}, \ldots, z_{k}\right)
$$

with

$$
W_{k}(.)=\int_{z_{d k}^{*}}^{z_{k}}\left(\chi^{\frac{1}{q_{k}}}-z_{d k}^{* \frac{1}{q_{k}}}\right)^{1+S-q_{k}} d \chi
$$

From the previous step, it can be observed that $W_{k}($.$) has the following properties:$

$$
\begin{aligned}
& \frac{\partial W_{k}(.)}{\partial z_{k}}=e^{1+S-q_{k}} \\
& \frac{\partial W_{k}(.)}{\partial z_{m}}=-\left(1+S-q_{k}\right) \frac{\partial z_{d k}^{* \frac{1}{q_{k}}}}{\partial z_{m}} \int_{z_{d k}^{*}}^{z_{k}}\left(\chi^{\frac{1}{q_{k}}}-z_{d k}^{\frac{1}{q_{k}}}\right)^{S-q_{k}} d \chi
\end{aligned}
$$

also, it is easy to show that $V_{k}($.$) is C^{1}$ and positive definite, which verifies $V_{k}()=.\leq$ $\max \left\{\frac{1}{1+S}, 2\right\} \sum_{m=1}^{k} e_{m}^{1+S}$. The time derivative of $V_{k}($.$) satisfies$ 
178 The inverted pendulum in control theory and robotics

$$
\begin{aligned}
\dot{V}_{k}(.) \leq & -(n-k+1+\kappa) \sum_{m=1}^{k-1} e_{m}^{2 S}+e_{k-1}^{1+S-q_{k-1}}\left(z_{k}-z_{d k}^{*}\right)+e_{k}^{1+S-q_{k}} z_{k+1} \\
& +e_{k}^{1+S-q_{k}} d_{k}(z)+\sum_{m=2}^{k-1} \frac{\partial W_{k}(.)}{\partial z_{m}} \dot{z}_{m}+\sum_{m=1}^{k-1} \tau_{m} \\
\leq & -(n-k+1+\kappa) \sum_{m=1}^{k-1} e_{m}^{2 S}+e_{k-1}^{1+S-q_{k-1}}\left(z_{k}-z_{d k}^{*}\right)+e_{k}^{1+S-q_{k}} z_{k+1} \\
& +\sum_{m=2}^{k-1} \frac{\partial W_{k}(.)}{\partial z_{m}} z_{m+1}+\left[e_{k}^{1+S-q_{k}} d_{k}(z)+\sum_{m=2}^{k-1} \frac{\partial W_{k}(.)}{\partial z_{m}} d_{m}(z)\right]+\sum_{m=1}^{k-1} \tau_{m}
\end{aligned}
$$

If the $k$ th lumped unmatched uncertainty is defined as $\tau_{k}=e_{k}^{1+S-q_{k}} d_{k}(z)+$ $\sum_{m=2}^{k-1} \frac{\partial W_{k}(.)}{\partial z_{m}} d_{m}(z)$, then $(8.50)$ rewrites:

$$
\begin{aligned}
\dot{V}_{k}(.) \leq & -(n-k+1+\kappa) \sum_{m=1}^{k-1} e_{m}^{2 S}+e_{k-1}^{1+S-q_{k-1}}\left(z_{k}-z_{d k}^{*}\right)+e_{k}^{1+S-q_{k}} z_{k+1} \\
& +\sum_{m=2}^{k-1} \frac{\partial W_{k}(.)}{\partial z_{m}} z_{m+1}+\sum_{m=1}^{k} \tau_{m}
\end{aligned}
$$

Next, we bound the second and the fourth terms of the right hand side of (8.51). First according to Lemma 8.2, it holds that

$$
\begin{aligned}
\left|e_{k-1}^{1+S-q_{k-1}}\left(z_{k}-z_{d k}^{*}\right)\right| & \leq 2^{1+S-q_{k-1}}\left|e_{k-1}\right|^{1+S-q_{k-1}}\left|e_{k}\right|^{q_{k}} \\
& \leq \frac{\left|e_{k-1}\right|^{2 S}}{2}+C_{k}\left|e_{k}^{2 S}\right|
\end{aligned}
$$

with $C_{k}$ a positive constant. As for the fourth term, it is easy to obtain the following:

$$
\left|\sum_{m=2}^{k-1} \frac{\partial W_{k}(.)}{\partial z_{m}} z_{m+1}\right| \leq\left(1+S-q_{k}\right) 2^{1-q_{k}}\left|e_{k}\right|^{S}\left|\sum_{m=2}^{k-1} \frac{\partial z_{d k}^{*}}{\partial z_{m}} z_{m+1}\right|
$$

To further bound the fourth term in (8.51), we need to conduct the analysis by inductive argument and assume that at step $k-1$, the following holds

$$
\left|\sum_{m=2}^{k-2} \frac{\partial z_{d k}^{*}}{\partial z_{m}} z_{m+1}\right| \leq \sum_{m=1}^{k-1} \gamma_{(k-1) m} e_{m}^{S}
$$

where $\gamma_{(k-1) m} \geq 0$, then show the inequality also holds for $k$. Therefore, we have

$$
\begin{aligned}
\left|\sum_{m=2}^{k-1} \frac{\partial z_{d k}^{*}}{\partial z_{m}} z_{m+1}\right| & \leq\left|-\beta_{k-1}^{\frac{1}{q_{k}}} \sum_{m=2}^{k-1} \frac{\partial e_{k-1}}{\partial z_{m}} z_{m+1}\right| \\
& \leq \beta_{k-1}^{\frac{1}{q_{k}}}\left|\frac{z_{k-1}^{\frac{1}{q_{k}}}-1}{q_{k-1}} z_{k}+\sum_{m=2}^{k-2} \frac{\partial z_{k-1}^{* \frac{1}{q_{k}-1}}}{\partial z_{m}} z_{m+1}\right|
\end{aligned}
$$


In light of the definition of the tracking error, it is worth recalling that $e_{m}=z_{m}^{\frac{1}{q_{m}}}-z_{m d}^{* \frac{1}{q_{m}}}$ and $z_{m}^{*}=-\beta_{m-1} e_{m-1}^{q_{m}}$, it can then be inferred that $z_{m}^{* \frac{1}{q_{m}}}=-\beta_{m-1}^{\frac{1}{q_{m}}} e_{m-1}$, and therefore, the following inequality holds

$$
\left|z_{m}\right| \leq\left|e_{m}+z_{m}^{* \frac{1}{q_{m}}}\right|^{q_{m}} \leq\left|e_{m}\right|^{q_{m}}+\beta_{m-1}\left|e_{m-1}\right|^{q_{m}}
$$

Applying (8.56) to the inequality (8.55) and using the assumption of the inequality (8.54) yields

$$
\begin{aligned}
\left|\sum_{m=2}^{k-1} \frac{\partial z_{d k}^{*}}{\partial z_{m}} z_{m+1}\right| \leq & \beta_{k-1}^{\frac{1}{q_{k}}}\left[\frac { 1 } { q _ { k - 1 } } ( | e _ { k - 1 } | ^ { 1 - q _ { k - 1 } } + \beta _ { k - 2 } ^ { \frac { 1 } { q _ { k - 1 } } - 1 } e _ { k - 2 } ^ { 1 - q _ { k - 1 } } ) \left(\left|e_{k}\right|^{q_{k}}\right.\right. \\
& \left.\left.+\beta_{k-1}\left|e_{k-1}\right|^{q_{k}}\right)+\sum_{m=2}^{k-2} \frac{\partial z_{d k}^{*}}{\partial z_{m}} z_{m+1}\right] \\
\leq & \sum_{m=1}^{k} \gamma_{k m}\left|e_{m}\right|^{S}
\end{aligned}
$$

with $\gamma_{k m}$ being a positive constant. Therefore,

$$
\begin{aligned}
\left|\sum_{m=2}^{k-1} \frac{\partial W_{k}(.)}{\partial z_{m}} z_{m+1}\right| & \leq\left(1+S-q_{k}\right) 2^{1-q_{k}}\left|e_{k}\right|^{S}\left(\sum_{m=1}^{k} \gamma_{k m}\left|e_{m}\right|^{S}\right) \\
& \leq \frac{1}{2} \sum_{m=1}^{k-1} e_{m}^{2 S}+\bar{C}_{k}\left|e_{k}\right|^{2 S}
\end{aligned}
$$

with $\bar{C}_{k} \geq 0$ being a positive constant.

Substituting (8.52) and (8.58) into (8.51) leads to

$$
\begin{aligned}
\dot{V}_{k}(.) \leq & -(n-k+1+\kappa) \sum_{m=1}^{k-1} e_{m}^{2 S}+\left(C_{k}+\bar{C}_{k}\right)\left|e_{k}\right|^{2 S}+\frac{\left|e_{k-1}\right|}{2}+\frac{1}{2} \sum_{m=1}^{k-1} e_{m}^{2 S} \\
& +\sum_{m=1}^{k} \tau_{k}+e_{k}^{1+S-q_{k}}\left(z_{k+1}-z_{d(k+1)}\right)^{*}+e_{k}^{1+S-q_{k}} z_{k+1}^{*} \\
\leq & -(n-k+\kappa) \sum_{m=1}^{k-1} e_{m}^{2 S}+\left(C_{k}+\bar{C}_{k}\right)\left|e_{k}\right|^{2 S}+e_{k}^{1+S-q_{k}}\left(z_{k+1}-z_{d(k+1)}^{*}\right) \\
& +e_{k}^{1+S-q_{k}} z_{k+1}^{*}+\sum_{m=1}^{k} \tau_{k}
\end{aligned}
$$

By introducing the virtual control $z_{(k+1) d}^{*}=-\beta_{k} e_{k}^{q_{k+1}}$ with $\beta_{k}$ selected such that $\beta_{k} \geq$ $n-k+\kappa+C_{k}+\bar{C}_{k}>0$, we get 
180 The inverted pendulum in control theory and robotics

$$
\dot{V}_{k}(.) \leq-(n-k+\kappa) \sum_{m=1}^{k} e_{m}^{2 S}+e_{k}^{1+S-q_{k}}\left(z_{k+1}-z_{(k+1) d}^{*}\right)+\sum_{m=1}^{k} \tau_{k}
$$

Step $n$ : This is the final stage of the design where the real control input appears in the dynamics. For this step, consider the $n$th part of the Lyapunov candidate function

$$
V_{n}\left(z_{1}, \ldots, z_{n}\right)=V_{n-1}(.)+W_{n}\left(z_{1}, \ldots, z_{n}\right)
$$

where

$$
W_{n}(.)=\int_{z_{n d}^{*}}^{z_{n}}\left(\chi^{\frac{1}{q_{n}}}-z_{n}^{\frac{1}{q_{n}}}\right)^{1+S-q_{n}} d \chi
$$

then, it is obvious to conclude that $V_{n}($.$) is C^{1}$, positive definite and satisfies $V_{n}($. $\max \left\{\frac{1}{1+S}, 2\right\} \sum_{m=1}^{n} e_{m}^{1+S}$. Furthermore, let $\widetilde{G}=G-\widehat{G}$ be the estimate error of the matched and unmatched uncertainty, and $G$ is the total lumped uncertainty to be defined later. From the above inductive argument, one can conclude that

$$
\begin{aligned}
\dot{V}_{n} \leq & -\kappa \sum_{m=1}^{n-1} e_{m}^{2 S}+e_{n}^{1+S-q_{n}} \dot{z}_{n}+\left(C_{n}+\bar{C}_{n}\right) e_{n}^{2 S}+\sum_{m=1}^{n-1} \tau_{m} \\
\leq & -\kappa \sum_{m=1}^{n-1} e_{m}^{2 S}+e_{n}^{1+S-q_{n}}\left(b_{n} u+d_{n}(z)\right)+\left(C_{n}+\bar{C}_{n}\right) e_{n}^{2 S} \\
& +e_{n}^{1+S-q_{n}}\left(e_{n}^{q_{n}-1-S} \sum_{m=1}^{n-1} \tau_{m}\right) \\
\leq & -\kappa \sum_{m=1}^{n-1} e_{m}^{2 S}+b_{n} e_{n}^{1+S-q_{n}} u+\left(C_{n}+\bar{C}_{n}\right) e_{n}^{2 S}+b_{n} e_{n}^{1+S-q_{n}}\left(\frac{d_{n}(z)}{b_{n}}\right. \\
& \left.+\frac{e_{n}^{q_{n}-1-S}}{b_{n}} \sum_{m=1}^{n-1} \tau_{m}\right)
\end{aligned}
$$

where $C_{n}>0$ and $\bar{C}_{n}>0$ are positive constants. The actual control law can therefore be designed as follows:

$$
u=-\beta_{n} e_{n}^{q_{n+1}}-\widehat{G} \operatorname{sign}(\sigma)
$$

with the compensator being designed as follows:

$$
\dot{\widehat{G}}=|\sigma|, \quad \sigma=e^{1+S-q_{n}}
$$

where $\widehat{G}$ is the estimation of the total uncertainty function $G$ defined as $G=$ $\frac{d_{n}(z)}{b_{n}}+\frac{e_{n}^{q_{n}-1-S}}{b_{n}} \sum_{m=1}^{n-1} \tau_{m}$, and $\beta_{n}$ is a positive design parameter chosen arbitrarily by the designer.

The main result of this chapter can be summarized in the following theorem. 


\section{Theorem 8.2}

Consider the $n$th order underactuated system in the $X$-space represented by (8.20), through the coordinate transformation (8.21) and under Assumptions 8.1 and 8.2, the finite-time convergence objective specified in (8.25) is achieved by the control input $u$ consisting of (8.64) and the compensator (8.65) with the virtual control $z_{m d}^{*}, m=1, \ldots, n$ being applied.

Proof. Consider the Lyapunov function $V=V_{n}($.$) . Since \frac{b_{n}}{2} \widetilde{G}^{2}$ is positive definite, then $V \leq V_{n}()+.\frac{b_{n}}{2} \widetilde{G}^{2}$. Taking the derivative of $V$ along (8.63), yields

$$
\begin{aligned}
\dot{V} \leq & \dot{V}_{n}-b_{n} \widetilde{G} \widehat{G} \\
\leq & -\kappa \sum_{m=1}^{n-1} e_{m}^{2 S}+b_{n} e_{n}^{1+S-q_{n}} u+\left(C_{n}+\bar{C}_{n}\right) e_{n}^{2 S}+b_{n} e_{n}^{1+S-q_{n}}\left(\frac{d_{n}(z)}{b_{n}}\right. \\
& \left.+\frac{e_{n}^{q_{n}-1-S}}{b_{n}} \sum_{m=1}^{n-1} \tau_{m}\right)-b_{n} \widetilde{G} \dot{\widehat{G}} \\
\leq & -\kappa \sum_{m=1}^{n-1} e_{m}^{2 S}+b_{n} e_{n}^{1+S-q_{n}} u+\left(C_{n}+\bar{C}_{n}\right) e_{n}^{2 S}+b_{n} e_{n}^{1+S-q_{n}} G-b_{n} \widetilde{G} \dot{G}
\end{aligned}
$$

Substituting the control law (8.64) and the update law (8.65) in (8.66) leads to

$$
\begin{aligned}
\dot{V} \leq & -\kappa \sum_{m=1}^{n-1} e_{m}^{2 S}-b_{n} \beta_{n} e_{n}^{1+S-q_{n}+q_{n+1}}+\left(C_{n}+\bar{C}_{n}\right) e_{n}^{2 S}-b_{n} \sigma \widehat{G} \operatorname{sign}(\sigma) \\
& +b_{n} \sigma G-b_{n} G \dot{\widehat{G}} \\
\leq & -\kappa \sum_{m=1}^{n-1} e_{m}^{2 S}-\left(b_{n} \beta_{n}-C_{n}-\bar{C}_{n}\right) e_{n}^{2 S}+b_{n}(G \sigma-\widehat{G}|\sigma|)-b_{n} G|\sigma|
\end{aligned}
$$

If $\beta_{n}$ is chosen such that $\beta_{n} b_{\max }-C_{n}-\bar{C}_{n}>\kappa$, it follows from (8.67) that

$$
\dot{V} \leq-\kappa \sum_{m=1}^{n} e_{m}^{2 S}
$$

Noting that $V=V_{n}(.) \leq \max \left\{\frac{1}{1+S}, 2\right\} \sum_{m=1}^{n} e_{m}^{1+S}$ and using Lemma 8.1, we have

$$
V_{n}(.)^{\frac{2 S}{1+S}} \leq \max \left\{\frac{1}{1+S}, 2\right\} \sum_{m=1}^{n} e_{m}^{2 S}
$$

Let $\alpha:=\frac{2 S}{1+S}$, then it is easy to conclude that

$$
\frac{d V(.)}{d t} \leq \mu V^{\alpha}
$$


182 The inverted pendulum in control theory and robotics

with $\mu=\frac{\kappa}{\max \left\{\frac{1}{1+S}, 2\right\}}$. From (8.70), it is straightforward to conclude from Theorem 8.1 that the solutions of the closed-loop system consisting of (8.24) and the control input (8.64) along with the compensator (8.65) are finite-time stable, and therefore, the limit in (8.25) is satisfied. This completes the proof.

\subsection{Numerical simulations: the case of the cart-pole system}

The cart-pole inverted pendulum depicted schematically in Figure 8.1 consists of a pole mounted on a cart by means of a pivot in such a way that the pole can freely swing in the $x-y$ plane. The equations of motion can be obtained by neglecting friction in the pivot and by applying the Euler-Lagrange formulation. The system model can be represented as follows [35]:

$$
\begin{aligned}
\left(M+m \sin ^{2} \theta\right) \ddot{x}-m \sin \theta\left(l \dot{\theta}^{2}-g \cos \theta\right) & =u \\
\left(M+m \sin ^{2} \theta\right) l \ddot{\theta}+m l \dot{\theta}^{2} \sin \theta \cos \theta-(M+m) g \sin \theta & =-\cos \theta(u)
\end{aligned}
$$

where $M$ is the mass of the cart, $m$ is the mass of the pendulum, $l$ is half the length of the pendulum, i.e., the distance from the pivot to the center of mass of the pendulum, $g$ is the acceleration due to gravity. The cart-pole system has two equilibrium points, one of which is known as the stable vertically downward position where $\theta=\pi$, and the other one being the unstable vertically upward position where $\theta=0 . F(t)$ is the horizontal force being applied to the cart to swing the pendulum until it reaches the desired unstable position where $\theta=0, \dot{\theta}=0$ and $\dot{x}=0$. As can be seen from the equations of motion (8.71), only one control $u$ appears in both dynamic equations of the cart-pole system; therefore, the whole system is underactuated. Meaning that it has fewer control inputs than degrees of freedom. Let $\mathbf{x}=[x, \dot{x}, \theta, \dot{\theta}]^{\top}=\left[x_{1}, x_{2}, x_{3}, x_{4}\right]^{\top} \in$ $\mathbb{R}^{4}$, then the state space representation of the cart-pole system becomes

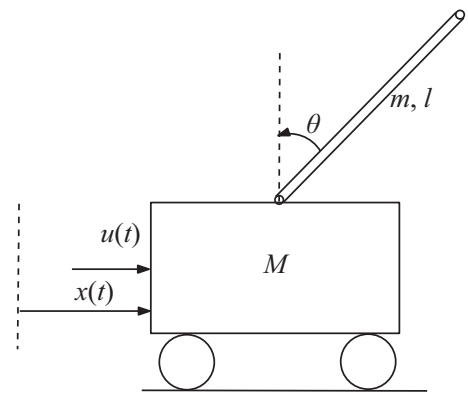

Figure 8.1 Schematic view of the cart-pole inverted pendulum 


$$
\begin{aligned}
& \dot{x}_{1}=x_{2} \\
& \dot{x}_{2}=f_{2}(\mathbf{x})+b_{2}(\mathbf{x}) u \\
& \dot{x}_{3}=x_{4} \\
& \dot{x}_{4}=f_{4}(\mathbf{x})+b_{4}(\mathbf{x}) u
\end{aligned}
$$

where $\quad f_{2}(\mathbf{x})=\frac{m x_{4}^{2} \sin x_{3}-m g \sin x_{3} \cos x_{3}}{M+m \sin ^{2} x_{3}}, \quad b_{2}(\mathbf{x})=\frac{1}{M+m \sin ^{2} x_{3}}, \quad f_{4}(\mathbf{x})=$ $\frac{(M+m) g \sin x_{3}-m l x_{4}^{2} \sin x_{3} \cos x_{3}}{M l+m l \sin ^{2} x_{3}}$ and $b_{4}(\mathbf{x})=\frac{-\cos x_{3}}{M l+m l \sin ^{2} x_{3}}$. The state space representation (8.72) is in the form (8.20) with $n=4$. The system can be readily transformed from the $X$-space into $Z$-space using the coordinate transformation (8.21) as follows:

$$
\begin{aligned}
& z_{1}=x_{1}-\int_{0}^{x_{3}} \frac{b_{2}(s)}{b_{4}(s)} d s=x_{1}+l \ln \left(\sec x_{3}+\tan x_{3}\right) \\
& z_{2}=x_{2}-\frac{b_{2}(\mathbf{x})}{b_{4}(\mathbf{x})} x_{4}=x_{2}+l x_{4} \sec x_{3} \\
& z_{3}=x_{3} \\
& z_{4}=x_{4}
\end{aligned}
$$

with the change of coordinate (8.73), the system dynamics in the $Z$-space becomes

$$
\begin{aligned}
& \dot{z}_{1}=z_{2}+d_{1}(z) \\
& \dot{z}_{2}=z_{3}+d_{2}(z) \\
& \dot{z}_{3}=z_{4}+d_{3}(z) \\
& \dot{z}_{4}=b_{4}(z) \mathbf{u}+d_{4}(z)
\end{aligned}
$$

where $d_{1}(z)=d_{3}(z)=0$ and

$$
\begin{aligned}
& d_{2}(z)=-z_{3}-l z_{4}^{2} \sec z_{3} \tan z_{3}+\frac{(M+m) g \tan z_{3}-m g \sin z_{3} \cos z_{3}}{M+m \sin ^{2} z_{3}} \\
& d_{4}(z)=\frac{(M+m) g \sin z_{3}-m l z_{4}^{2} \sin z_{3} \cos z_{3}}{M l+m l \sin ^{2} z_{3}} \\
& b_{4}(z)=\frac{-\cos z_{3}}{M l+m l \sin ^{2} z_{3}}
\end{aligned}
$$

The objective is then to control the transformed system (8.74) such that all states $z_{1}, z_{2}, z_{3}$ and $z_{4}$ converge in finite time to zero regardless of the internal uncertainties and external disturbances. For such system, the virtual controllers and the actual 
184 The inverted pendulum in control theory and robotics
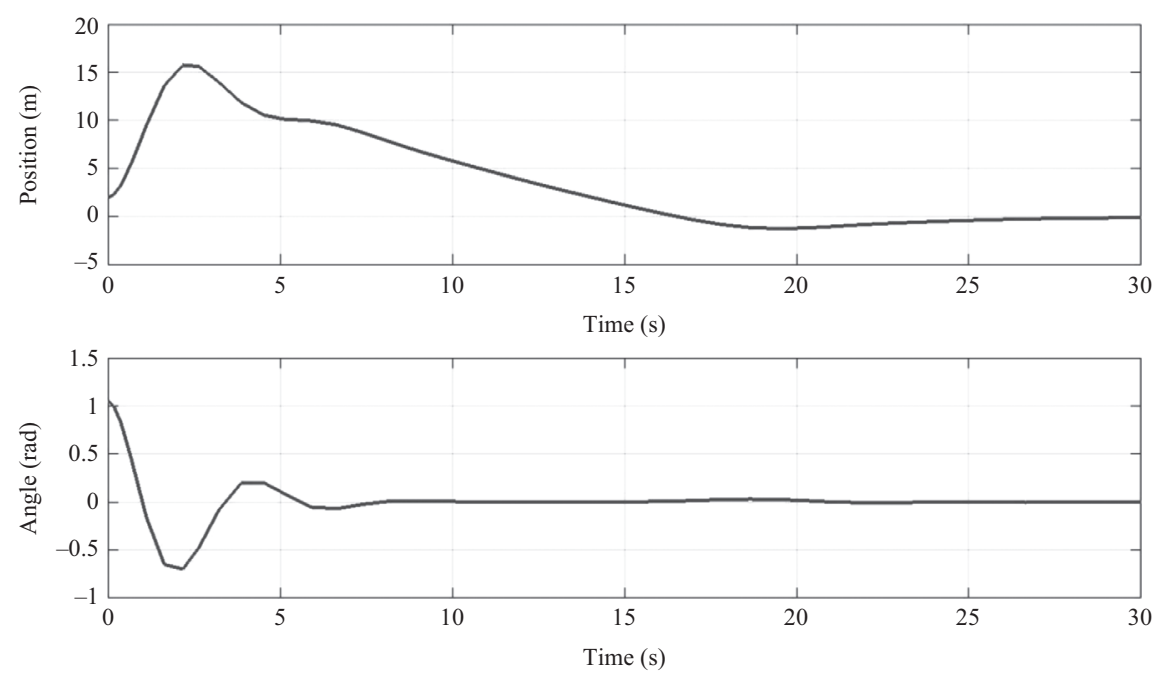

Figure 8.2 Trajectories of the cart position and pendulum angle versus time

control law are designed according to the development of Theorem 8.2 and are as follows:

$$
\begin{cases}z_{1 d}^{*}=0, & e_{1}=z_{1}^{\frac{1}{q_{1}}}-z_{1 d}^{* \frac{1}{q_{1}}} \\ z_{2 d}^{*}=-\beta_{1} e_{1}^{q_{2}}, & e_{2}=z_{2}^{\frac{1}{q_{2}}}-z_{2 d}^{* \frac{1}{q_{2}}} \\ z_{3 d}^{*}=-\beta_{2} e_{2}^{q_{3}}, & e_{3}=z_{3}^{\frac{1}{q_{2}}}-z_{3 d}^{* \frac{1}{q_{2}}} \\ z_{4 d}^{*}=-\beta_{3} e_{3}^{q_{4}}, & e_{4}=z_{4}^{\frac{1}{q_{2}}}-z_{4 d}^{* \frac{1}{q_{2}}}\end{cases}
$$

and

$$
\left\{\begin{array}{l}
u=-\beta_{4} e_{4}^{q_{5}}-\widehat{\widehat{G}} \operatorname{sign}(\sigma) \\
\dot{\widehat{G}}=|\sigma|, \quad \sigma=e^{1+S-q_{4}}
\end{array}\right.
$$

To demonstrate the performance of the proposed robust finite control law, we have performed numerical simulations on $\mathrm{MATLAB}^{\circledR} /$ Simulink $^{\circledR}$ framework, considering the following system parameters $m=1 \mathrm{~kg}, M=2 \mathrm{~kg}$ and $l=0.5 \mathrm{~m}$. The initial conditions are chosen as $\mathbf{x}=\left[1,0, \frac{\pi}{3}, 0\right]^{\top}$, the desired state is $\mathbf{x}_{d}=[0,0,0,0]^{\top}$. The control gains are chosen to be $\kappa=10, \beta_{1}=13, \beta_{2}=12, \beta_{3}=11$ and $\beta_{4}=20$. Different simulation scenarios have been considered. The first simulation scenario is the control of uncertain cart-pole system without external disturbances. The second one considers the case of an uncertain cart-pole system with the presence of external disturbances.

1. Scenario 1: Uncertain cart-pole system without external disturbances

In this scenario, we assume that the system dynamics is completely unknown except for the sign condition in Assumption 8.2. The obtained simulation results 


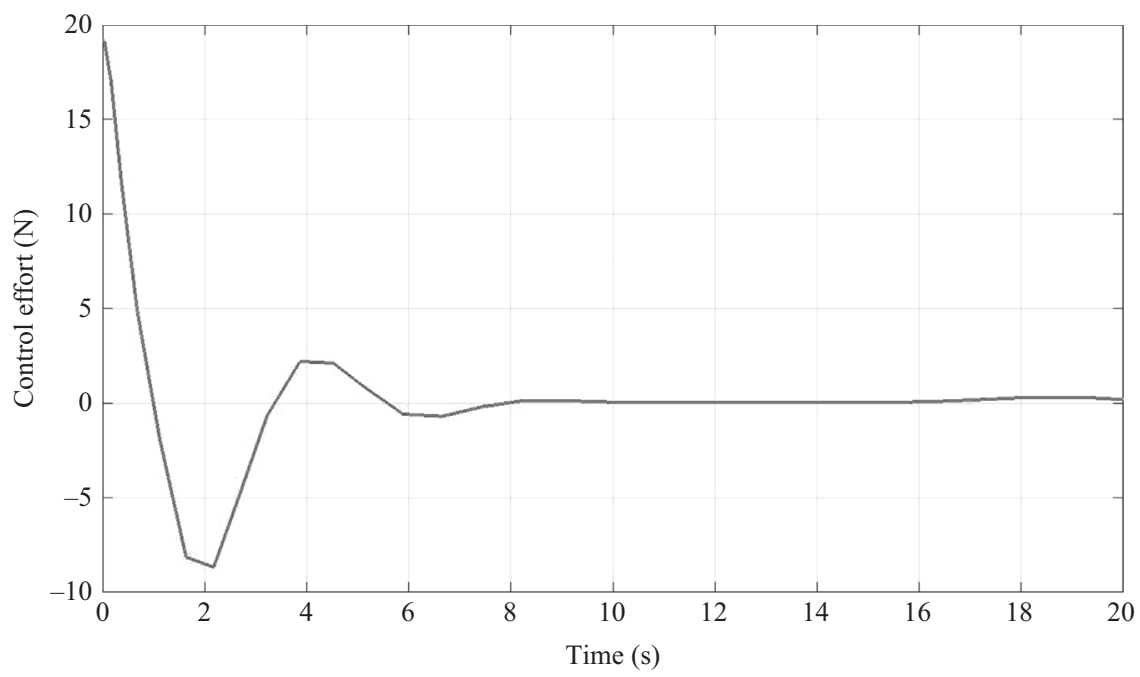

Figure 8.3 Evolution of the control effort versus time
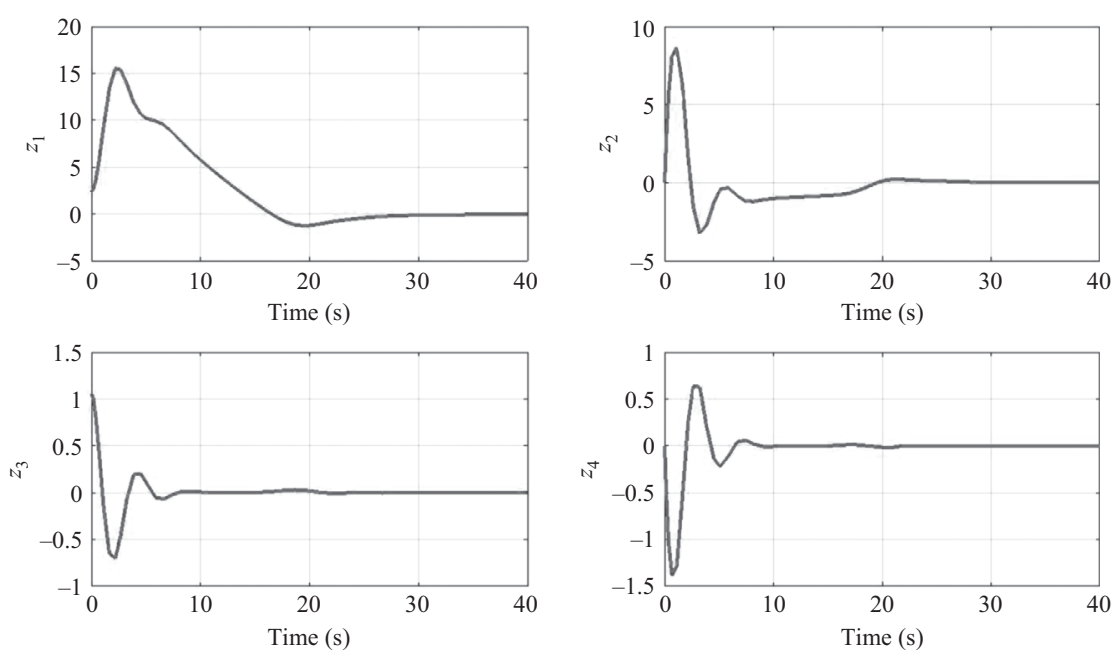

Figure 8.4 Evolution of the trajectories of Z-space state variables versus time

are shown in Figures 8.2-8.4. It is clear in Figure 8.2 how the cart displacement and the pendulum angle converge in finite time to zero despite the uncertainties present in the system dynamics. The control effort being deployed is shown in Figure 8.3. Figure 8.4 shows the convergence to zero of the state variables in the $Z$-space. Clearly, the algorithm performs well in the presence of model parameters' uncertainties. 
186 The inverted pendulum in control theory and robotics
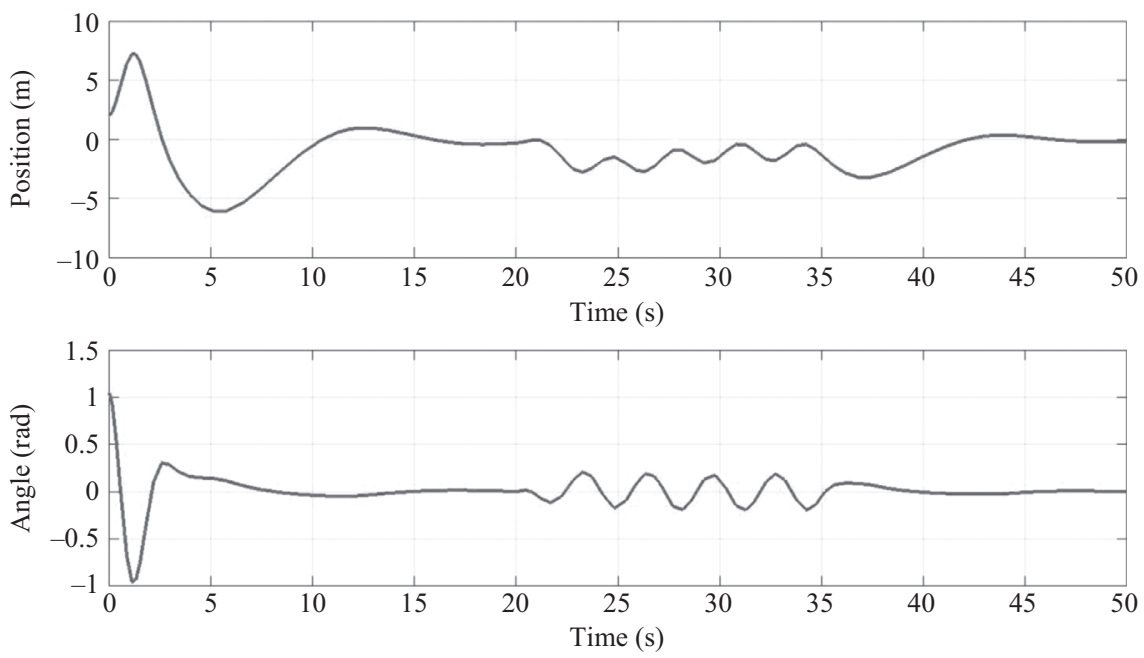

Figure 8.5 Evolution of the trajectories of the cart position and pendulum angle accounting for disturbances

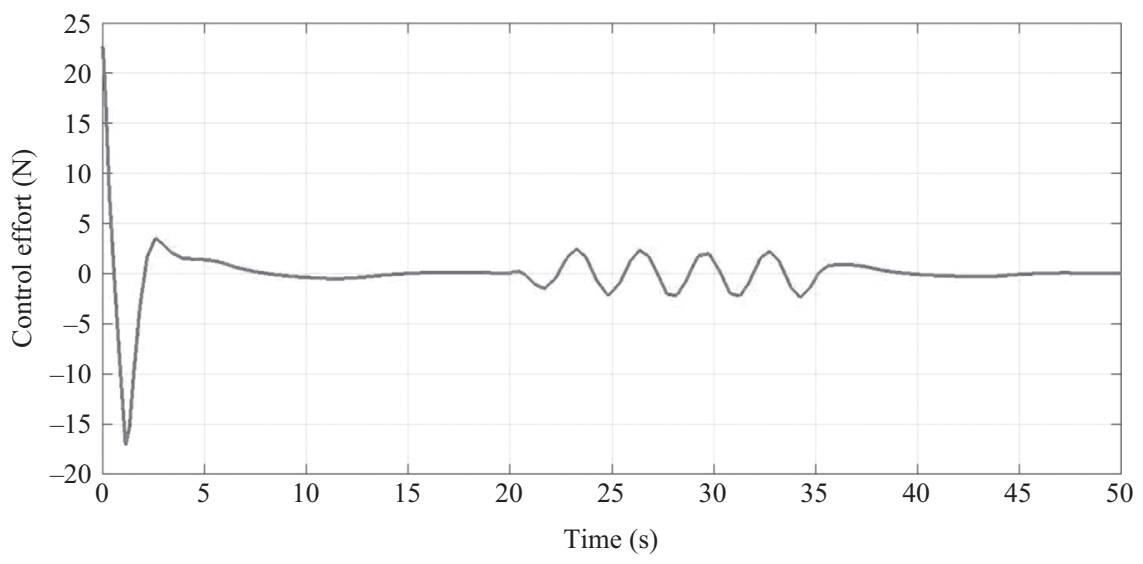

Figure 8.6 Evolution of the control effort accounting for disturbances

2. Scenario 2: Uncertain cart-pole system with external disturbances

In this simulation scenario, on top of the uncertainties that the system dynamics contains, a periodic perturbation $d(t)=\sin (2 t)$ is added as an external excitation to the cart-pole system during the time interval $t \in[20,35] \mathrm{s}$ in order to test the robustness of the proposed control approach. The obtained simulation results are depicted in Figures 8.5-8.7. From Figure 8.5, it can be noticed that despite the existing uncertainties and the considered external disturbance, 

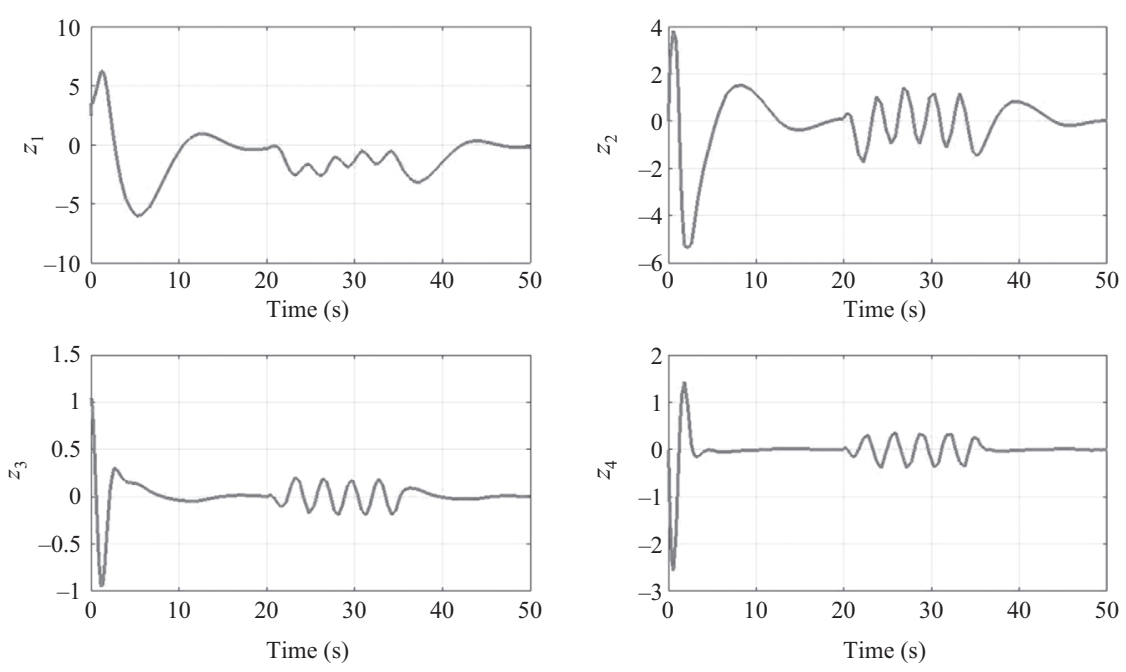

Figure 8.7 Evolution of the trajectories of Z-space state variables accounting for disturbances

the controller is able to steer the system to its desired values. The control effort after the application of the disturbances is shown in Figure 8.6, clearly the amplitude of the control input even with the external perturbation remains with reasonable size. The convergence of the state variables in $Z$-space is shown in Figure 8.7. From these figures, it can be seen that our control design is robust to tolerate significant variation of the system parameters as well as the external disturbances.

\subsection{Conclusion}

In this paper, the problem of global finite-time stabilization was addressed for the control of a class of uncertain underactuated mechanical systems. By integrating a fractional power feedback control method with a compensator, the uncertainties in the system can be effectively handled and finite-time stabilization is achieved. Future work is to extend the current design technique to a class of chained form systems perturbed by external disturbances as well as experiments on the inertia wheel inverted pendulum.

\section{References}

[1] N. Adhikary and C. Mahanta. 'Integral backstepping sliding mode control for underactuated systems: swing-up and stabilization of the cart-pendulum system', ISA Transactions, 2013, 58, pp. 870-880. 
188 The inverted pendulum in control theory and robotics

[2] F. Amato, M. Ariola, and P. Dorato. 'Finite-time control of linear systems subject to parametric uncertainties and disturbances', Automatica, 2001, 37, 9, pp. 1459-1463.

[3] S. Andary, A. Chemori, and S. Krut. 'Control of the underactuated inertia wheel inverted pendulum for stable limit cycle generation', RSJ Advanced Robotics, 2009, 23, 15, pp. 1999-2014.

[4] S. Andary and A. Chemori. 'A dual model-free control of non-minimum phase systems for generation of stable limit cycles', In Proc. 50th IEEE Conf. Decision Contr. (CDC'11), Montreal, Canada, 2011, pp. 1387-1392.

[5] S. Andary, A. Chemori, M. Benoit, and J. Sallantin. 'A dual model-free control of underactuated mechanical systems, application to the inertia wheel inverted pendulum', In American Control Conference (ACC'12), Montreal, Canada, 2012, pp. 1029-1034.

[6] M. Bergerman. Dynamics and Control of Underactuated Manipulators. PhD Thesis, Carnegie Mellon University, 1996.

[7] S. P. Bhat and D. S. Bernstein. 'Finite-time stability of continuous autonomous systems', SIAM Journal on Control and Optimization, 2000, 38, 3, pp. 751-766.

[8] W. Blajer and K. Kolodziejczyk. 'Modeling of underactuated mechanical systems in partly specified motion', Journal of Theoretical and Applied Mechanics, 2008, 46, 2, pp. 383-394.

[9] S. A. Bortoff and M. W. Spong. 'Pseudolinearization of the acrobot using spline functions', In Proc. 31st IEEE Conf. Decision Contr., Tucson, Arizona, USA, 1992, pp. 593-598.

[10] A. Chemori and M. Alamir. 'Nonlinear predictive control of under-actuated mechanical systems, application: the ECP 505 inverted pendulum', In MTNS'06 Proc. Mathematical Theory of Networks and Systems, Leven, Belgium, 2004.

[11] N. Khraief, A. Chemori and S. Belghith. ' External disturbance rejection in IDA-PBC controller for underactuated mechanical systems: from theory to real-time experiments', In IEEE Multi-Conference on Systems and Control (MSC'14), Antibes, France, 2014, pp. 1747-1752.

[12] D. Cheng and W. Lin. 'On normal form of nonlinear systems', IEEE Transactions on Automatic Control, 2003, 48, 11, pp. 1242-1248.

[13] A. Choukchou-Braham, B. Cherki, M. Djemai, and K. Busawon. Analysis and Control of Underwatcuated Mechanical Systems. London, UK: Springer, 2014.

[14] I. Fantoni and R. Lozano. Non-linear Control for Underactuated Mechanical Systems. New York, USA: Springer-Verlag, 2001.

[15] T. I. Fossen. Nonlinear Modelling and Control of Underwater Vehicles. PhD Thesis, Norwegian Institute of Technology, 1991.

[16] L. B. Freidovich, P. La Hera, U. Mettin, A. Robertsson, and A. S. Shiriaev. 'Shaping stable periodic motions of inertia wheel pendulum: theory and experiments', Asian Journal of Control, 2009, 11, 5, pp. 548-556. 
[17] L. Freidovicha, A. Robertssona, A. Shiriaeva, and R. Johanssonb. 'Periodic motions of the pendubot via virtual holonomic constraints: theory and experiments', Automatica, 2008, 44, pp. 785-791.

[18] S. Ge and F. Lewis. Autonomous Mobile Robots. CRC Press, 2006.

[19] V. T. Haimo. 'Finite-time controllers', SIAM Journal on Control and Optimization, 1986, 24, pp. 760-770.

[20] Y. Hong. 'Finite-time stabilization and stabilizability of a class of controllable systems', System \& Control Letters, 2002, 46, pp. 231-236.

[21] Y. Hong, J. Wang, and Z. Xi. 'Stabilization of uncertain chained form systems within finite settling time', IEEE Transactions on Automatic Control, 2005, 50, 9, pp. 1379-1384.

[22] Y. G. Hong and Z. P. Jiang. 'Finite-time stabilization of nonlinear systems with parametric and dynamic uncertainties', IEEE Transactions on Automatic Control, 2006, 51, pp. 1950-1956.

[23] Y. G. Hong, K. Wang, and D. Cheng. 'Adaptive finite-time control of nonlinear systems with parametric uncertainty', IEEE Transactions on Automatic Control, 2006, 51, pp. 858-862.

[24] X. Huang, W. Lin, and B. Yang. 'Global finite-time stabilization of a class of uncertain nonlinear systems', Automatica, 2005, 41, pp. 881-888.

[25] X. Huang, W. Lin, and B. Yang. 'Global finite-time stabilization of a class of uncertain nonlinear systems', Automatica, 2005, 41, 5, pp. 881-888.

[26] S. Jadlovska and J. Sarnovsky. 'Application of the state-dependent riccati equation method in nonlinear control design for inverted pendulum systems', In IEEE 11th International Symposium on Intelligent Systems and Informatics (SISY'13), pages 209-214, Subotica, Serbia, 2013.

[27] S. Jadlovska and J. Sarnovsky. 'A complex overview of modeling and control of the rotary single inverted pendulum system', Power Engineering and Electrical Engineering, 2013, 11, 2, pp. 73-85.

[28] S. Jadlovska and J. Sarnovsky. 'Modeling and optimal control of nonlinear underactuated mechanical systems a survey', In 13th Scientific Conference of Young Researchers FEI TU of Kosice, Herlany, Slovakia, 2013, pp. 32-35.

[29] N. Khalid and A. Y. Memon. 'Output feedback control of a class of underactuated nonlinear systems using extended high gain observer', Arabian Journal for Science and Engineering, 2016, 41, 9, pp. 3531-3542.

[30] H. Khalil. Nonlinear Systems. Prentice Hall, Upper Saddle River, Second ed., NJ, USA, 1996.

[31] K. Lee and V. 'Coverstone-Carroll. Control algorithms for stabilizing underactuated robots'. Journal of Robotic Systems, 1998, 15, 12, pp. 681-697.

[32] A. De Luca, R. Mattone, and G. Oriolo. 'Stabilization of underactuated planar 2r manipulator', International Journal of Robust and Nonlinear Control, 2000, 10, 4, pp. 181-198.

[33] Y. Nakamura, T. Suzuki, and M. Koinuma. 'Nonlinear behavior and control of nonholonomic free-joint manipulator', IEEE Transactions on Robotics and Automation, 1997, 13, 6, pp. 853-862. 
190 The inverted pendulum in control theory and robotics

[34] R. Reza Olfati-Saber. Nonlinear Control of Underactuated Mechanical Systems with Application to Robotics and Aerospace Vehicles. PhD Thesis, Massachusetts Institute of Technology, 2001.

[35] M. Park and D. Chwa. 'Swing-up and stabilization control of inverted pendulum systems via coupled sliding-mode control method', IEEE Transactions on Industrial Electronics, 2009, 56, pp. 3541-3555.

[36] G. Poulin, A. Chemori, and N. Marchand. 'Minimum energy oriented global stabilizing control of the PVTOL aircraft', International Journal of Control, 2007, 80, 3, pp. 430-442.

[37] C. Qian and W. Lin. 'A continuous feedback approach to global strong stabilization of nonlinear systems', IEEE Transactions on Automatic Control, 2001, 46, 7, pp. 1061-1079.

[38] D. Qian, J. Yi, and D. Zhao. 'Hierarchical sliding mode control for a class of SIMO under-actuated systems', Control and Cybernetics, 2008, 37, 1, pp. $1-18$.

[39] M. T. Ravichandran and A. D. Mahindrakar. 'Robust stabilization of a class of underactuated mechanical systems using time scaling and Lyapunov redesign', IEEE Transactions on Industrial Electronics, 2011, 58, pp. 4299-4313.

[40] M. Reyhanoglu, S. Ludvigsen, and O. Egeland. 'Discontinuous feedback control of a special class of underactuated mechanical systems', International Journal of Robust and Nonlinear Control, 2000, 10, 4, pp. 265-282.

[41] M. Spong. 'Partial feedback linearization of underactuated mechanical systems', In EEE/RSJ/GI International Conference on Intelligent Robots and Systems, Munich, Germany, 1994, pp. 314-321.

[42] M. Spong. 'The swing up control problem for the acrobot', IEEE Control Systems Magazine, February 1995, 15, 1, pp. 49-55.

[43] M. Spong. 'Energy based control of a class of underactuated mechanical system', In 13th IFAC World Congress, San Francisco, USA, 1996, pp. 431-436.

[44] M. Spong. Underactuated mechanical systems. Control Problems in Robotics and Automation, B. Siciliano and K. P. Valavanis Eds., LNCIS, Springer Verlag, 230:135-150, 1998.

[45] M. W. Spong and L. Praly. Control Using Logic-Based Switching, volume 222 of Lecture Notes in Control and Information Sciences, chapter Control of underactuated mechanical systems using switching and saturation, pages 162-172. Springer, Heidelberg, Germany, 2006.

[46] N. Touati and A. Chemori. 'Predictive control for the stabilization of a fast mechatronic system: from simulation to real-time experiments', In, Hangzhou, China, 2013International IFAC Symposium on Mechatronic Systems (Mechatronics'13), pp. 237-242.

[47] J. Xu, Z. Guo, and T. H. Lee. 'Design and implementation of integral sliding mode control on an underactuated two-wheeled mobile robot', ISA Transactions, 2013, 58, pp. 870-880. 
Chapter 8

\section{Finite-time stabilization of underactuated mechanical systems}

\section{Author Queries}

AQ1: As per style, the running heads should be of 65 characters (including space). Hence, we have shortened the title of the chapters in the book, wherever needed, so as to match the requirement. Please confirm if these shortened titles are fine.

AQ2: An extra closing parenthesis has been deleted in Equation (8.59). Please check, and correct if necessary.

AQ3: As Equation (8.68) contains label without equation content, we have removed the equation number and renumbered remaining equations. Please check and amend if necessary.

AQ4: Please supply the name of the city of publication for Reference [18].

AQ5: References [24] and [25] are almost similar. Please check and correct if necessary. 